\title{
ON THE NORMALIZER OF A GROUP IN THE CAYLEY REPRESENTATION
}

SURINDER K. SEHGAL

\author{
Department of Mathematics \\ The Ohio State University \\ Columbus, Ohio 43210
}

(Received October 21,1987 )

\begin{abstract}
If $G$ is embedded as a proper subgroup of $X$ in the Cayley representation of $G$, then the problem of "if $N_{X}(G)$ is always larger than $G$ " is studied in this paper.
\end{abstract}

KEY WORDS AND PHRASES: Cayley representation, Wreath product, permutation group. 1980 A.M.S. SUBJECT CLASSIFICATION CODE. 20B35, 20D25,

Let $R$ be the Cayley representation (i.e., the right regular representation) of a group $G$ given by $R(g)=\left(\begin{array}{c}x \\ x g\end{array}\right)$ for all $g \in G$ and $x \in G$. Under the mapping $R$, the group $G$ is embedded into a subgroup $R(G)$ of the symmetric group $S_{\Omega}$, the group of permutations on the set $\Omega$ consisting of the elements of the group $G$. We identify $R(G)$ with $G$ and say that $G$ is a subgroup of $S_{\Omega}$. The centralizer of $G$ in $S_{\Omega}$ consists precisely of the elements of the form $\left(\begin{array}{l}x \\ g x\end{array}\right)$. (See Lemma 1.) In particular, if $G$ is abelian then $G$ is self centralizing in $S_{\Omega}$. Also, the normalizer of $G$ in $S_{\Omega}$ is equal to $G \cdot \operatorname{Aut}(G)$ where $\operatorname{Aut}(G)$ is the full automorphism group of $G$ (see Lemma 2).

Suppose that the group $G$ is nonabelian. If $X$ is a subgroup of $S_{\Omega}$, containing a permutation of the type $\left(\begin{array}{c}x \\ g x\end{array}\right)$ for sme $g \in G-Z(G)$ such that the property

$$
G \lessgtr X \leq S_{\Omega}
$$

holds, then it follows that $N_{X}(G)$ contains $G$ properly. However, it is easy to see that any element of $S_{\Omega}$ which normalizes $G$ is not always a permutation of the form $\left(\begin{array}{l}x \\ g x\end{array}\right)$.

When the group $G$ is abelian, the permutations $\left(\begin{array}{l}x \\ g x\end{array}\right)$ all lie in $G$ and so $G$ is self centralizing in $S_{\Omega}$. In this way one cannot find a group $X$ satisfying $\left({ }^{*}\right)$ by the above method. However, $P$. Bhattacharya [1] proved that if $G$ is any finite, abelian $p$ group satisfying $(*)$ then $N_{X}(G) \geqslant G$. P. Bhattacharya and N. Mukherijee [2] also prove that if $G$ is any finite, nilpotent, Hall subgroup of $\boldsymbol{X}$ satisfying $\left(^{*}\right)$ and the Sylow $\boldsymbol{p}$ subgroups of $G$ are regular for all primes $p$ dividing the order of $G$, then $N_{X}(G) \supsetneqq G$. In other words, that $X$ must contain an element of the outer automorphism group of $G$. 
In this paper we will prove that if $G$ is any abelian Hall subgroup of $\boldsymbol{X}$, satisfying the condition $\left.{ }^{*}\right)$ then $G \lesseqgtr N_{X}(G)$. We will also give an example to show that the condition of being Hall subgroup is necessary in the above theorem. We will also show that if $G$ is any nilpotent, Hall subgroup of $X$ satisfying the condition $\left(^{*}\right)$ and the Sylow $p$ subgroups $P$ of $G$ do not have a factor group that is isomorphic to the Wreath product of $\left.Z_{p}\right\} Z_{p}$ then $G \lesseqgtr N_{X}(G)$. In particular it follows that if $G$ is any finite $p$-group and does not have a factor group isormophic to $Z_{p} \nmid Z_{p}$ then $G \lessgtr N_{X}(G)$ [i.e., the condition being a Hall subgroup is not necessary]. As a corollary it also follows that if $G$ is any regular $p$-group satisfying the conition $\left(^{*}\right)$ then $G \lessgtr N_{X}(G)$. We will give an example to show that the condition of $G$ having no factor group isomorphic to $Z_{p}\left\{Z_{p}\right.$ is necessary.

Lemma 1. Let $R$ be the right regular representation of a finite group $G$ and $L$, the left regular representation of $G$. Under the mappings $L$ and $R$, the groups $L(G)$ and $R(G)$ are subgroups of $S_{\Omega}$ and $C_{S_{\Omega}}(R(G))=L(G)$.

Proof: Let $\left(\begin{array}{l}x \\ x g\end{array}\right) \in R(G),\left(\begin{array}{l}x \\ h x\end{array}\right) \in L(G)$

$$
\begin{aligned}
\left(\begin{array}{c}
x \\
x g
\end{array}\right)\left(\begin{array}{c}
x \\
h x
\end{array}\right) & =\left(\begin{array}{c}
x \\
x g
\end{array}\right)\left(\begin{array}{c}
x g \\
h x g
\end{array}\right)=\left(\begin{array}{c}
x \\
h x g
\end{array}\right) \\
& =\left(\begin{array}{c}
x \\
h x
\end{array}\right)\left(\begin{array}{c}
h x \\
h x g
\end{array}\right)=\left(\begin{array}{c}
x \\
h x
\end{array}\right)\left(\begin{array}{c}
x \\
x g
\end{array}\right) .
\end{aligned}
$$

Hence $L(G) \subseteq C_{S_{\Omega}}(R(G))$.

Now suppose $\left(\begin{array}{c}x \\ x^{\prime}\end{array}\right) \in S_{\Omega}$ and $\left(\begin{array}{c}x \\ x^{\prime}\end{array}\right)$ centralizes $R(G)$. So $\left(\begin{array}{c}x \\ x g\end{array}\right)\left(\begin{array}{l}x \\ x\end{array}\right)=\left(\begin{array}{c}x \\ x g\end{array}\right)\left(\begin{array}{c}x g \\ (x g)\end{array}\right)=\left(\begin{array}{c}x \\ (x g)\end{array}\right)$ and $\left(\begin{array}{l}x \\ x^{\prime}\end{array}\right)\left(\begin{array}{c}x \\ x g\end{array}\right)=\left(\begin{array}{c}x \\ x^{\prime}\end{array}\right)\left(\begin{array}{c}x^{\prime} \\ x^{\prime} g\end{array}\right)=\left(\begin{array}{c}x \\ x^{\prime} g\end{array}\right)$.

Since $\left(\begin{array}{c}x \\ x^{\prime}\end{array}\right) \in C_{S_{\Omega}}$, so $x^{\prime} g=(x g)^{\prime}$ for all $x, g \in G$.

Hence $x^{\prime}=(x g)^{\prime} g^{-1}$. Now plug in $g=x^{-1}$. So $x^{\prime}=1^{\prime} \cdot x$. Thus $\left(\begin{array}{c}x \\ x^{\prime}\end{array}\right)=\left(\begin{array}{c}x \\ 1^{\prime} \cdot x\end{array}\right) \in L(G)$. Hence $C_{S_{\Omega}}(R(G))=L(G)$.

Lemma 2: With the same notation as in Lemma 1, we have $N_{S_{\Omega}}(R(G))=R(G) \cdot A u t(G)$.

Proof: Let $\left(\begin{array}{l}x \\ x^{\prime}\end{array}\right) \in \operatorname{Aut}(G)$ then $\left(\begin{array}{l}x \\ x^{\prime}\end{array}\right) \in S_{\Omega}$,

$$
\begin{aligned}
\left(\begin{array}{c}
x \\
x^{\prime}
\end{array}\right)^{-1}\left(\begin{array}{c}
x \\
x g
\end{array}\right)\left(\begin{array}{c}
x \\
x^{\prime}
\end{array}\right) & =\left(\begin{array}{c}
x^{\prime} \\
x
\end{array}\right)\left(\begin{array}{c}
x \\
x g
\end{array}\right)\left(\begin{array}{c}
x g \\
(x g)^{\prime}
\end{array}\right) \\
& =\left(\begin{array}{c}
x^{\prime} \\
(x g)^{\prime}
\end{array}\right)=\left(\begin{array}{c}
x^{\prime} \\
x^{\prime} g^{\prime}
\end{array}\right)=\left(\begin{array}{c}
x \\
x g^{\prime}
\end{array}\right) \in R(G) .
\end{aligned}
$$

Hence $A u t(G) \subseteq N_{S_{\Omega}}(R(G))$. Conversely, let $\left(\begin{array}{c}x \\ x^{\prime}\end{array}\right)$ be an arbitrary element of $N_{S_{\Omega}}(G)$. Let $a=1^{\prime}$. So $\left(\begin{array}{c}x \\ x a^{-1}\end{array}\right) \in R(G)$. Let $O=\left(\begin{array}{c}x \\ x^{\prime}\end{array}\right)\left(\begin{array}{c}x \\ x a^{-1}\end{array}\right)$. So 0 sends 1 to 1 . Now $\left(\begin{array}{c}x \\ x\end{array}\right)^{-1}\left(\begin{array}{l}x \\ x g\end{array}\right)\left(\begin{array}{l}x \\ x\end{array}\right) \in$ $R(G)$. So $\left(\begin{array}{c}x^{0} \\ x\end{array}\right)\left(\begin{array}{c}x \\ x g\end{array}\right)\left(\begin{array}{c}x g \\ (x g)^{0}\end{array}\right)=\left(\begin{array}{c}x^{0} \\ (x g)^{0}\end{array}\right)=\left(\begin{array}{c}x^{0} \\ x^{0} g^{*}\end{array}\right)$ since it lies in $R(G)$, i.e., $(x g)^{0}=x^{0} \cdot g^{*}$. Plug in $x=1$, we get $g^{*}=g^{0} \Rightarrow(x g)^{0}=x^{0} \cdot g^{0} \Rightarrow 0$ is an autormophism of $G \Rightarrow$ $N_{S_{\Omega}}(R(G))=R(G) \cdot A u t(G)$.

Lemma 3: Let $G$ be any finite group satisfying the condition (*). Then for any $\alpha \in \Omega$

(i) $G \cap X_{\alpha}=\{e\}$.

(ii) $\boldsymbol{X}=\boldsymbol{G} \cdot \boldsymbol{X}_{\boldsymbol{\alpha}}$

(iii) $\boldsymbol{X}_{\boldsymbol{\alpha}}$ is core free, i.e., it does not contain any non-identify normal subgroup of $\boldsymbol{X}$.

Proof: Recall that here $G$ is identified with $R(G)$ in $G \leq X \leq S_{\Omega}$. Since $R$ is the right regular representation of $G$, so $R(g)$ does not fix any $\alpha \in \Omega$ except when $g=e$. So $G \cap X_{\alpha}=$ 
$\{e\}$. Also $X$ acts transitively on $\Omega,\left|\alpha^{X}\right|=|\Omega|=|G|$. Now $\left[X: X_{\alpha}\right]=\left|\alpha^{X}\right|=|G|$. So $X=G \cdot X_{\alpha}$. For part (iii) suppose $N \triangleleft X$ and $N \subseteq X_{\alpha}$. So $N \subseteq \cap_{x \in X^{-1} X_{\alpha} x \text {, i.e., }}$ if $n$ is an arbitrary element of $N$, then $n$ can be written as $n=x^{-1} u x$ for all $x \in X$ and some $u \in X_{\alpha}$. Here $u$ depends on $x$, i.e., $x \cdot n=u \cdot x$ or $\alpha^{x n}=\alpha^{u x}=\alpha^{x}$ since $u$ fixes $\alpha$, i.e., $n$ fixes $\alpha^{x}$ for all $x \in X$, but $X$ acts transitively on $\Omega \Rightarrow n$ fixes every element of $X \Rightarrow n=e \Rightarrow N=\{e\}$.

Lemma 4: (Core Theorem): Let $H$ be any subgroup of $G$ with $[G: H]=n$. then $G /$ core $H$ is isomorphic to a subgroup of $S_{n}$ where core $H$ is the largest normal subgroup of $G$ which is contained in $H$.

Proof: Let $\Omega$ be the set of distinct right cosets of $H$ in $G$, i.e., $\Omega=\left\{H g_{1}, H g_{2}, \ldots, H g_{n}\right\}$. Then the mapping $\sigma$ defined by $\sigma(g)=\left(\begin{array}{c}H g_{i} \\ H g_{i} g\end{array}\right)$ is a transitive permutation representation of $G$ of degree $n$ with Kernel of $\sigma=$ core $H$.

Theorem 5: Let $G$ be a finite abelian, Hall subgroup of $X$, satisfying the condition $\left(^{*}\right)$. Then $N_{X}(G) \supsetneqq G$.

Proof: Suppose the result is false, i.e., there exists a subgroup $X$ of $S_{\Omega}$ satisfying $G \lessgtr X \leq$ $S_{\Omega}$ and $N_{X}(G)=G$. Amongst all subgroups of $S_{\Omega}$ containing $G$ property, pick $X$ to be smallest. In other words, $G$ is a maximal subgroup of $X$. Let $|G|=p_{1}^{\alpha_{1}}, p_{2}^{\alpha_{2}}, \cdots, p_{t}^{\alpha_{t}}$ with $p_{i}$ distinct primes. Let $P_{i}$ be Sylow $p_{i}$ subgroups of $G$ for $i=1,2, \ldots, t$. Since $G$ is a maximal subgroup of $X$, so $N_{X}\left(P_{i}\right)=G$ or $N_{X}\left(P_{i}\right)=X$. Renumber the $p_{i}$ 's if necessasry and say $N_{X}\left(P_{i}\right)=G$ for $i=1, \ldots, \ell$ and $N_{X}\left(P_{i}\right)=X$ for $i=\ell+1, \ldots, t$. For $i=1, \ldots, \ell$, $N_{X}\left(P_{i}\right)=C_{X}\left(P_{i}\right)=G$. So by Burnside Lemma $X$ has a normal $p_{i}$ complement. For $j=$ $\ell+1, \ldots, t, P_{j} \triangleleft X \Rightarrow C_{X}\left(P_{j}\right) \triangleleft X G \subseteq C_{X}\left(P_{j}\right) \Rightarrow C_{X}\left(P_{j}\right)=X=N_{X}\left(P_{j}\right)$. So $X$ has a normal $P_{i}$ complement $M_{i}$ for all $i \Rightarrow X_{\alpha}=\bigcap_{i=1}^{t} M_{i}, X_{\alpha} \triangleleft S$ which is a contradiction to Lemma 3.

In the case where $G$ is abelian, but not Hall subgroup of $\boldsymbol{X}$, the result is not true as illustrated by the following example.

Example 6: Let $X=Z_{3} \times S_{3}=\langle a\rangle \times\left\langle b, c \mid b^{3}=c^{2}=1, c^{-1} b c=b^{-1}\right\rangle$.

Let $G=Z_{3} \times Z_{2}=\langle a\rangle \times\langle c\rangle \simeq Z_{6}$. Let $H$ be the subgroup of $X$ of order 3 generated by the ordered pair $(a, b)$. Then $H$ is not normal in $X$ since $(e, c)$ does not normalize $H$. So $H$ is core free, of index 6 in $X$. By Lemma $4, G \lessgtr X \leq S_{6}$. Now $G$ is abelian, not Hall subgroup of $X$ and $N_{X}(G)=G$.

Theorem 7: $\quad$ Let $G$ be a finite, nilpotent, Hall subgroup of $X$, satisfying the condition $\left({ }^{*}\right)$. Suppose that the Sylow $p$ subgroups $P$ of $G$ do not have a factor group isomorphic to the Wreath product of $Z_{p} \backslash Z_{p}$ for all primes $p$ dividing the order of $G$. Then $N_{X}(G) \supsetneqq G$.

Proof: Suppose the result is false, i.e., there exists a subgroup $X$ of $S_{\Omega}$ satisfying $G \lessgtr X \leq$ $S_{\Omega}$ and $N_{X}(G)=G$. Amongst all subgroups of $S_{\Omega}$ containing $G$ properly, pick $X$ to be smallest. In other words $G$ is a maximal subgroup of $X$. Let $|G|=p_{1}^{\alpha_{1}} \cdot p_{2}^{\alpha_{2}} \cdot \ldots \cdot p_{t}^{\alpha_{t}}$, here $p_{i}$ are all distinct primes. Since $G$ is nilpotent, so $G=P_{1} \times P_{2} \times \ldots \times P_{t}$ where $P_{i}$ are Sylow $p_{i}$ subgroups of $G$. So we have either $N_{X}\left(P_{i}\right)=G$ or $N_{X}\left(P_{i}\right)=X$. Renumber the $p_{i}$ 's if necessary and say $N_{X}\left(P_{i}\right)=G$ for $i=1, \ldots \ell$ and $N_{X}\left(P_{i}\right)=X$ for $i=\ell+1, \ldots, t$.

Let us look at the case $i=1, \ldots, \ell$. We have $N=N_{X}\left(P_{i}\right)=G$. By Yoshida's transfer theorme [3], $X$ has normal $p_{i}$ complement $M_{i}$.

Let $M=\cap_{i=1}^{\ell} M_{i}$. So $p_{i} \backslash|M|$ for $i=1, \ldots, \ell$. Now for $j=\ell+1, \ldots, t, N_{X}\left(P_{j}\right)=X$. So $P_{j} \triangleleft X$ which implies that $C_{X}\left(P_{j}\right) \triangleleft X$ and $P_{j} C_{X}\left(P_{j}\right) \triangleleft X$ and $G \subseteq P_{j} \cdot C_{X}\left(P_{j}\right) \Rightarrow$ $P_{j} C_{X}\left(P_{j}\right)=X$. 
For $\alpha \in \Omega$, by Lemma $3 X=G \cdot X_{\alpha} ; G \cap X_{\alpha}=1 ;\left(|G|,\left|X_{\alpha}\right|\right)=1 \Rightarrow X_{\alpha} \subseteq$ $C_{X}\left(P_{j}\right) \cap M \Rightarrow X_{\alpha} \subseteq C_{M}\left(P_{j}\right)$ for $j=\ell+1, \ldots, t .|M|=p_{j+1}^{\alpha_{j+1}} \ldots p_{t}^{\alpha_{t}} \cdot\left|X_{\alpha}\right| \Rightarrow$ $X_{\alpha} \Delta M \Rightarrow X_{\alpha}$ is a characteristic subgroup of $M \Delta G \Rightarrow X_{\alpha} \Delta G$, which is a contradiction to Lemma 3.

As an immediate corollary to the theorem, we get the result of P. Bhattacharya and N. Mukherjee [2].

Corollary 8: Let $G$ be a finite, regular $p$ subgroup of $X$ and satisfies the condition (*), then $N_{X}(G) \gtreqless G$.

Proof: If $G$ is not a Hall subgroup of $X$ then $G$ is propertly contained in a Sylow $p$ subgroup of $X$ and so $N_{X}(G) \supsetneqq G$. So we can assume that $G$ is a Hall subgroup of $X$. Now $G$ being a regular $p$ group $\Rightarrow G$ does not have a factor group isomorphic to $Z_{p}\left\{Z_{p}\right.$. So Theorem 7 proves the result.

Corollary 9. Let $G$ be a finite, nilpotent, Hall subgroup of $X$, satisfying the condition $\left(^{*}\right)$. Suppose further that Sylow $p$ subgroups of $G$ are regular for all primes $p$ dividing the order of $G$ then $N_{X}(G) \supsetneqq G$.

Corollary 10: Let $G$ be a finite $p$ group, satisfying the condition (*). Suppose that $G$ does not have a factor group isomorphic to $\left.Z_{p}\right\} Z_{p}$, then $G \lessgtr N_{X}(G)$.

The condition that the Sylow $p$ subgroups of $G$ in Theorem 6 have the property that it has no homomorphic isomorphic to $Z_{p}\left\{Z_{p}\right.$ is necessary. Se example below.

Example: Let $X$ be the simple group of order 168. Let $G \in S y l_{2}(X)$. Then $G \cong Z_{2} \backslash Z_{2}$ so $G$ is nilpotent, Hall subgroup of $X$. Since $H=$ the normalizer of a Sylow 7 subgroup has index 8, so by Lemma 4, $G \subseteq X \subseteq S_{8}$, i.e., $G$ satisfies the condition (*) but $N_{X}(G)=G$.

\section{References}

1. P. Bhattacharya, On the normalizer of a group in the Cayley representation, Bull. Austrian Math. Soc. 25 (1982) 81-84.

2. P. Bhattacharya and N. Mukherjee, On the normalizer of a subgroup of a finite group and the Cayley embedding; J. of Pure and Applied Algebra 33 (1984) 253-257.

3. T. Yoshida, Character theoretic transfer; J. of Algebra 52, (1978) 1-38.

4. B. Huppert, Endliche Gruppen I (Springer, New York, 1967).

5. M. ISSACS, Character Theory of Finite Groups. (Academic Press 1976).

6. H. Zassenhaus, The Theory of Groups (Chelsea, 1956). 


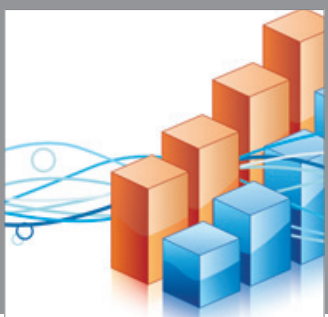

Advances in

Operations Research

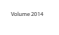

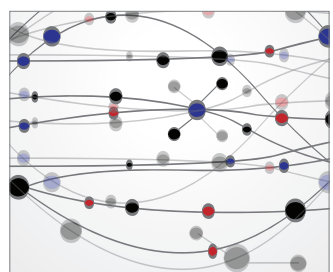

\section{The Scientific} World Journal
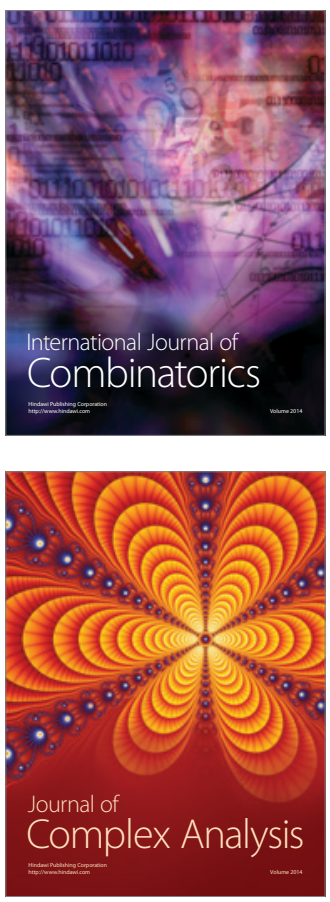

International Journal of

Mathematics and

Mathematical

Sciences
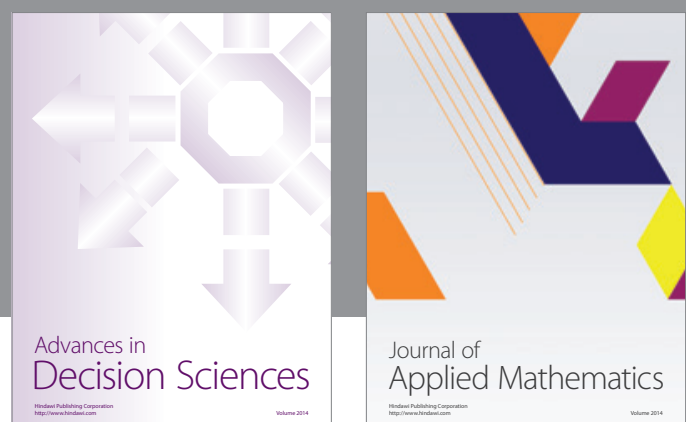

Journal of

Applied Mathematics
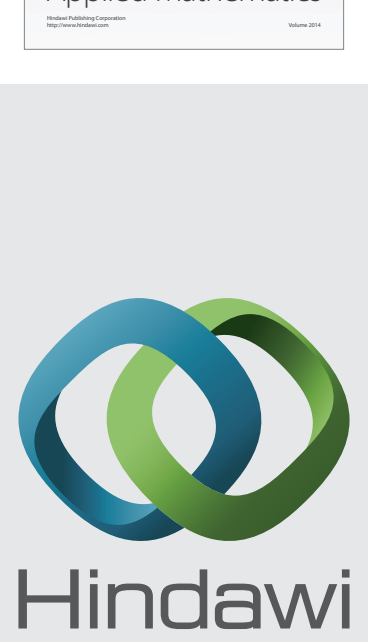

Submit your manuscripts at http://www.hindawi.com
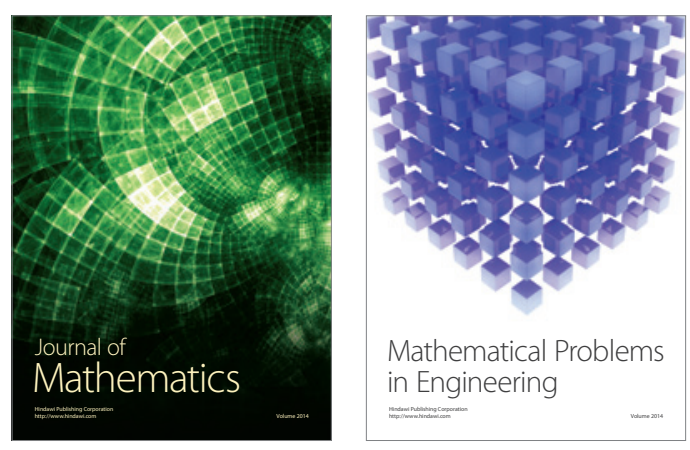

Mathematical Problems in Engineering
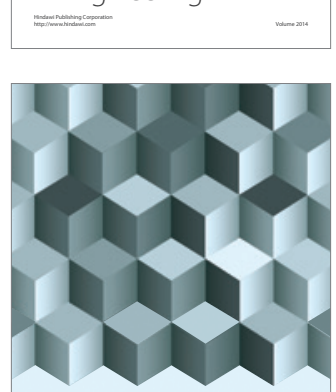

Journal of

Function Spaces
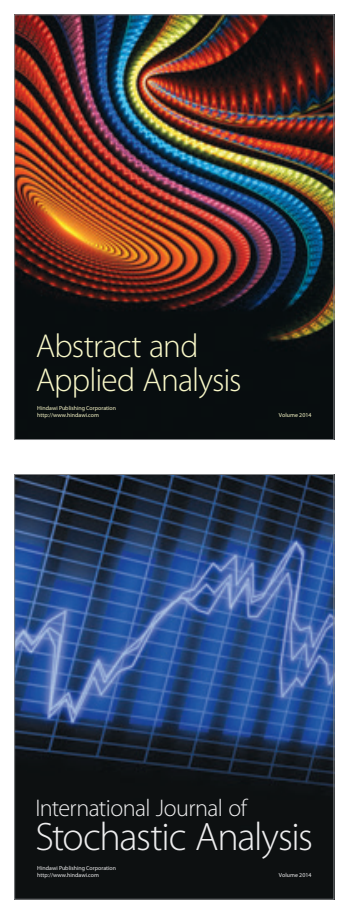

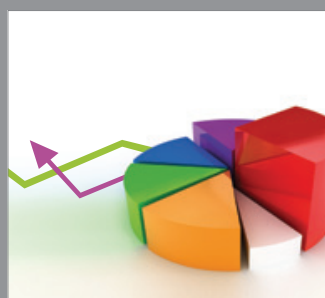

ournal of

Probability and Statistics

Promensencen
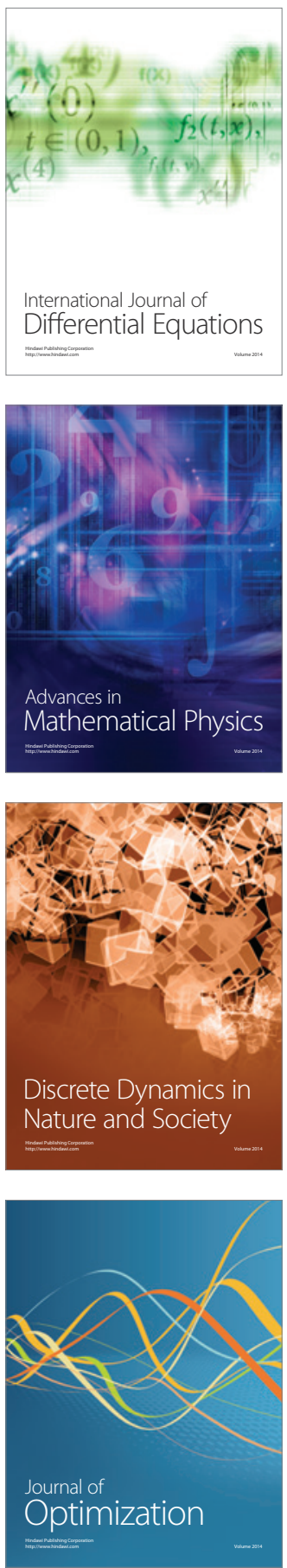